\title{
Item concreteness vs spatial organization as predictors of visual imagery*
}

\author{
BRIAN BYRNE $\dagger$ \\ McMaster University, Hamilton, Ontario, Canada
}

\begin{abstract}
The relative contributions of item concreteness and interitem spatial organization to recall processes were studied by attempting to induce modality-specific interference between recall and response. Separate groups of $12 \mathrm{Ss}$ learned lists of items that varied in physical or referential visual characteristics. They later signaled information about them sither vocally or via a visually guided response. Some ways of presenting lists for learning that are traditionally regarded as increasing reliance on mediating imagery were effective in generating conflict between recall and the visually guided response. This effectiveness was limited to presentation conditions and list types that introduced spatial organization into the stimulus material. The concreteness of individual items was not useful in predicting visual conflict.
\end{abstract}

It seems apparent that visual imagery plays an important role in learning and memory and that items high in concreteness frequently arouse imagery. This paper investigates whether there may not be another characteristic of stimulus material that can supplement or even override concreteness in predicting that material will be represented in imaginal form. This characteristic is spatial organization.

Authors often appeal to common sense experience in discussing visual imagery, and often in these anecdotes spatial aspects figure large. Consider this example from Paivio's recent book (1971): "Occasionally, when I have to list the names of my colleagues from memory, I have found myself visualizing the hallways in which their offices are located, systematically moving past these offices, then picturing and naming the occupants [p. 3]." A well-known example is a request for the reader to enumerate the windows in his house (Shepard, 1966; Neisser, 1968). Most people report that they have to imaginally move around the house, visualizing and counting the windows. In both of these examples, there seems to be a distinction between generating images of things (colleagues, windows) and engaging in a spatial search, a directional activity (moving along a hallway or around a house) that links the more static images. Skinner (1953) makes reference to a similar kind of distinction when he talks of discriminative and manipulative responses of private seeing, and Berlyne (1965) refers to situational and transformational thinking, relating the distinction to visual imagery. Paivio (1971) characterizes the division as being between static and dynamic representational processes.

*This research was supported by a grant from the National Research Council of Canada (APA210) to L. R. Brooks. Parts of this work were presented at the annual conventions of the Psychonomic Society, San Antonio, 1970, and the Australian Psychological Society, Canberra, 1972.

TThis report is based on a PhD dissertation presented to the School of Graduate Studies, McMaster University. The help of L. Brooks during all stages of the work and of P. Sheehan and W. Noble in the preparation of the manuscript is gratefully acknowledged. The author is now at the University of New England, Armidale, N.S.W. 2351, Australia.
This hypothesized dynamic component, usually viewed as operating on spatial aspects of the memorized material, has received little attention in the experimental study of imagery and its role in learning. Generally, in manipulating imagery level in memory experiments, investigators have concentrated on characteristics of items considered singly. For example, words are grouped together as being high in imagery because each one has, individually, been rated as evoking imagery readily. What remains unexplored is whether item concreteness alone is the best indicator that material may be stored in visual form. Items are not usually grouped with potential for spatial integration.

Consider, by way of illustration, the following two word lists: List 1-pig trunk dog fire wheat child bale insect ocean tulip; List 2-chimney roof ceiling wall carpet floor door steps path lawn. Both contain highly concrete items and pilot work revealed that people asked to learn them as serial lists frequently reported visualizing the words' referents. But in List 2 , Ss additionally reported a feeling of "moving down" an integrated imaginal structure. Using concreteness of the single items as the sole predictor of how the two word groups are represented internally, little difference would be expected. But on the assumption that spatial cohesiveness exists among the referents of List 2 and that this quality induces an additional dynamic component in visual imagery, differences would be expected. Certainly Ss' reports of their strategies seemed consistent with this latter expectation.

How might these ideas be tested? Traditionally, clues as to how material is represented in memory have been gained by varying some stimulus characteristic and examining effects on acquisition and recall. In the long run, however, this approach relies on the E's ability to select differentially memorable sets of material, where the difference in learning rates can only be attributed to the hypothetically important variable. How difficult this can be is illustrated by Kintsch's (1972) recent demonstration that the concreteness-abstractness dimension has often been confounded with lexical complexity and that this latter variable can account for 


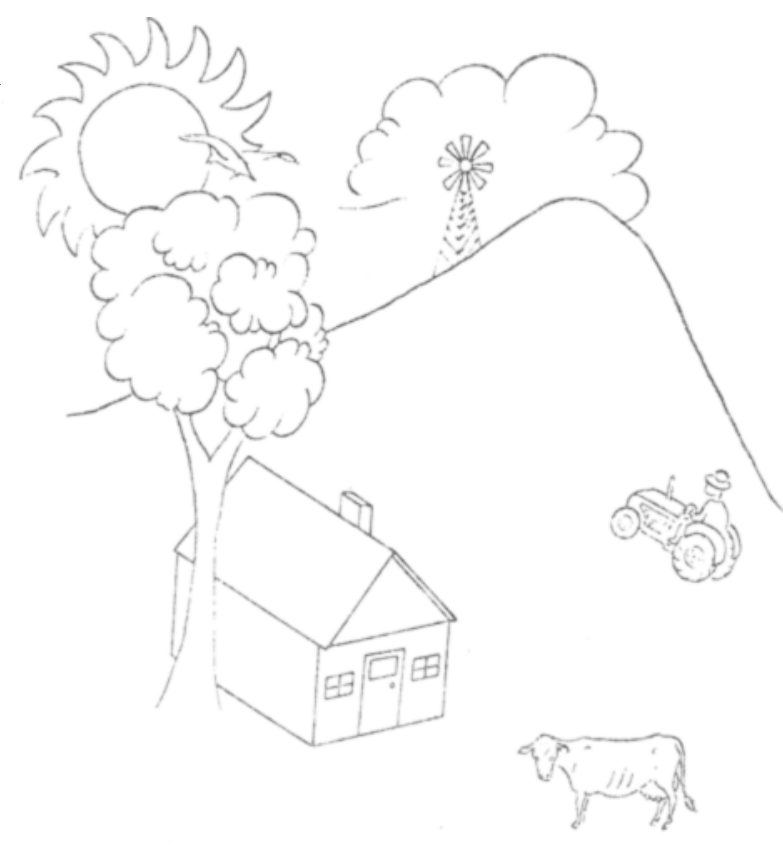

Fig. 1. Scene for FARM list.

some of the variation attributed to concreteness.

A technique for studying internal visual representation that probably avoids these difficulties is to attempt to demonstrate that the acquisition or recall of certain kinds of stimulus material is disrupted by concurrent visual perception. At base is the assumption that visual imagery and visual perception share processing capacity (see Brooks, 1967, 1968, 1970; Segal \& Fusella, 1970 for supporting evidence). Conflict between memory functions and perceptual activity may be taken as evidence that the $S$ is storing (or attempting to store) the material in imaginal form.

Such a technique was adopted for the present series of experiments and was an adaptation of one used earlier by Brooks (1968). The Ss were asked to memorize a list of words such as one of those presented above and then, from memory, to successively categorize each item for the presence or absence of a certain characteristic (e.g., does the word refer to an animal). They were given two ways of responding, saying "yes" or "no" for each item in turn or pointing to a column of Ys and Ns printed on a card before them. If recall of the word list requires visual imagery, then the visually guided pointing response should disrupt recall.

The first two experiments might be regarded as an attempt to validate this experimental technique. The Ss learned a word list under conditions widely regarded as likely to induce visual imagery (with pictorial support in Experiment I and under instructions to visualize in Experiment II). The responses of both groups were compared with those of another group that learned the same list in a way less likely to generate mediating imagery. If the method is to be useful, evidence of greater recall disruption should be found with the first two groups than with the last mentioned. In the second part of the experimental series, attention was turned to the major question-whether spatial organization produces evidence of visual imagery over and above that attributable to item concreteness.

\section{EXPERIMENT I}

Method

\section{Subjects}

Two independent groups of $12 \mathrm{McM}$ aster University students acted as paid volunteers ( $\$ 2$ per hour). The Ss were assigned randomly to groups with the restriction that there were six males and six females in each group.

\section{Material}

A list of 10 nouns, code named FARM, was used: sun bird tree house cow tractor farmer hill windmill cloud. A scene was drawn (Fig. 1) in which these 10 objects figured in a counterclockwise sequence.

\section{Procedure}

The procedure breaks into three main parts: (1) introduction to and practice in categorizing words in a memorized list, (2) acquisition of the FARM list, and (3) timed categorization trials, with FARM as the memorized material. The two groups of Ss were treated differently only in the acquisition phase.

In demonstrating the categorization task, $\mathrm{E}$ first placed the list snow dog train boy bus before $S$ and informed him that he could be asked to say whether or not each word referred to an animal. The E produced the sequence "no yes no yes no" to illustrate. When $S$ understood the nature of the task, he was asked to say whether or not each word referred to a man-made object. (This vocal response will be referred to as VOC). Following this, E demonstrated the pointing response (called V-G for visually guided). He signaled the appropriate response for the category animal by pointing to a response sheet in the fashion indicated in Fig. 2. The $S$ demonstrated his understanding by categorizing for man-made in the same way. At this point, $S$ was required to memorize a (new) list of words (mouse chair rock cat table) to a criterion of three consecutive errorless recall trials and then to

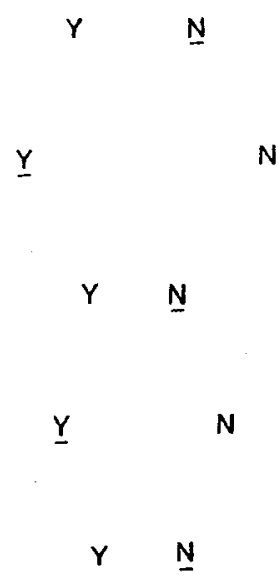

Fig. 2. Form of response sheet for visually guided response. Letters underlined are those that $E$ pointed to during introduction to categorizing. In the main part of the experiments, a similar 10-line sheet was used. Pointing was done with a pen. 
PREDICTORS OF VISUAL IMAGERY

categorize the items from memory. A total of four of these practice categorization trials were given, two for animal and two for man-made, with the response types VOC V-G V-G VOC used in that order. This practice procedure was then repeated with another five-word list.

The acquisition phase will now be described for the group given the scene as pictorial support (FARMSCENE group). The $\mathrm{E}$ placed the scene in Fig. 1 before $\mathrm{S}$, telling him that it constituted the material to be learned. The $\mathrm{E}$ pointed to and named the items. in the order shown above, at the rate of three items per $2 \mathrm{sec}$. This procedure was repeated, following which $S$ twice named the items in order, still looking at the scene. The picture was then removed and $S$ attempted an ordered recall of IV the list. Each time a mistake was made, $\mathrm{S}$ was again shown the scene and asked to name the items. If he was correct, he was immediately required to recall the words again, to a criterion of three successive errorless recall trials.

At this point, E illustrated how to use a starting point other than sun by placing the scenc before $S$ and naming the entire list, beginning with house and ending with tree. The scene was again removed and $S$ was required to recall the list from another starting point (e.g., hill). In all, four more recall trials were given, each with a different starting point. The reason for this aspect of the acquisition phase was that in the main part of the experiment (categorization of the list items from memory). different starting points were used to minimize the possibility that $\mathrm{Ss}$ would simply remember a string of yes and no responses and, thus, be able to respond without actually mentally running through the items.

The acquisition procedure for the other group (F:ARMSPOKEN) differed only in that $S$ at no stage saw the scene. The E simply read the list four times at initial presentation and once following each erroneous recall trial.

In preparation for the categorization trials, $S$ s were told that animal and man-made were to be the bases of categorization and that the positive instances of the former were bird cow farmer and of the latter house tractor windmill. They were also told to proceed as quickly as possible during categorization, with the stipulation that accuracy was important. Prior to each list categorization, Ss were told $(a)$ the response mode for that trial (VOC or V-G). (b) the categorization basis (animal or man-made). and (c) the starting point. They were then free to start their responses. Timing began at the end of the last instruction (starting point) and continued until $S$ produced 10 yes and no responses or reached the last line of the output sheet. depending on response mode.

Response modes were alternated in pairs (two VOC. two V-G. etc.). Categorization bases were randomized, with the restriction that each occurred five times in 10 trials. Each word in the list served as a starting point as often as every other and in random order within that restriction. This part of the experiment continued until $S$ produced 14 errorless categorization trials with each response mode. seven for each categorization basis 128 in all). For the purposes of alternating response modes, no distinction was made between correct and crroneous responsey. Sometimes $\mathrm{S}$ would indicate that he could go no further in a

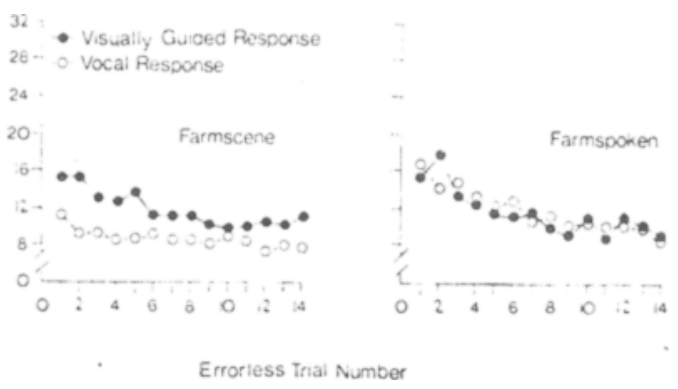

Fig. 3. Response speeds by trials. Experiment I.
Table 1

Mean Response Speeds (Seconds) for Each Experimental Group*

\begin{tabular}{|c|c|c|c|}
\hline \multirow{2}{*}{$\begin{array}{l}\text { Experiment } \\
\text { and Condition }\end{array}$} & \multicolumn{2}{|c|}{ Response Speed } & \\
\hline & $V-G$ & VOC & \\
\hline FARMSCENE & 11.7 & 8.5 & $(.65)$ \\
\hline FARMSPOKEN & 11.7 & 11.8 & $(.52)$ \\
\hline $\begin{array}{l}\text { FARMSPOKEN with } \\
\text { Imagery Instructions }\end{array}$ & 13.4 & 11.7 & $(.45)$ \\
\hline FARMSTACK & 13.3 & 12.8 & $(.57)$ \\
\hline HOUSE & 12.3 & 10.7 & $(.44)$ \\
\hline PIGMATRIX & 19.2 & 14.7 & $(.81)$ \\
\hline $\begin{array}{l}\text { Abstract Word } \\
\text { Matrix }\end{array}$ & 14.7 & 11.8 & $(.82)$ \\
\hline
\end{tabular}

*Standard error of mean within-S differences ( $V-G$ minus $V O C$ ) in parentheses.

Note-Slight discrepancies between response speeds in this table and mean within-S differences reported in text are due to rounding off.

trial, and it was counted as an error. A short rest was given about halfway through this phase. and at the end Ss were questioned on their coding strategies and response mode preferences.

\section{Results and Discussion}

During the acquisition phase, the FARMSCENE group took fewer trials to reach criterion $(3.4$; minimum 3$)$ than did FARMSPOKEN Ss [4.7. $\mathrm{t}(22)=2.18$, $\mathrm{p}<.025]$. This result was consistent with the claim that pictures aid learning via the generation of visual imagery. Note, however, that Ss were not under any explicit pressure to learn rapidly and, thus, little weight can be at tached to acquisition data.

The result of major interest is the categorization response speeds; these are shown in Fig. 3 and Table 1. For the group shown the scene during acquisition (FARMSCENE), there was a clear difference between the two response modes at output, a difference of $3.2 \mathrm{sec}$ favoring the vocal response (VOC). (In the treatment of results, no distinction is made between response times for the two categorization bases, animal and man-made.) This mean within-S difference (V-G - VOC) was significantly greater than the corresponding figure of $-0.2 \mathrm{sec}$ for $\mathrm{Ss}$ in FARMSPOKEN $[\mathrm{t}(22)=4.00, \mathrm{p}<.001]$.

A comment needs to be made at this point about the logic of interpretation of these response speeds. Absolute latencies are difficult to interpret, and absolute differences (V.G - VOC) similarly so. For example, it would be tempting to treat zero response mode differences as meaning that one method of responding did not disrupt recall more than the other. But at this stage. there is no reason to claim that. because it is not known what the "true" difference would be if, in fact. neither response mode disrupted recall at all. It could be an actual time difference in one direction or the other. What is sought. therefore, is an interaction between treatments (in this case. acquisition with and without pictorial support) and within-s response mode 
differences, such that a greater difference exists in the case of one treatment group than another. This is, of course, exactly what has occurred in this experiment. Taking the $-0.2-\mathrm{sec}$ difference for FARMSPOKEN as a basis for comparison (a figure which, perhaps coincidentally, does not differ significantly from zero), it can be seen that in FARMSCENE Group V-G disrupted recall more, relative to any disruption due to VOC. There were signs, in other words, of conflict between recall and the visually guided response in FARMSCENE that were not present in FARMSPOKEN, suggesting that $S s$ in the former group were relying more heavily on internal visual representation during recall.

This interpretation of the results fits neatly with Ss' own comments. All FARMSCENE Ss reported using a mental picture of the scene during recall for categorization, an image derived from the picture presented to them during acquisition. Many spontaneously reported a directional component in their imagery, a sense of "going around" the scene mentally. Furthermore, some made comments like the following: "I couldn't see it [the image] as well when I was pointing-I couldn't move my eyes back and forth on the page [response sheet] and back and forth on the [imagined] picture." In contrast, Ss in FARMSPOKEN emphasized verbal strategies. There was some imagery reported, mostly of item subgroups, such as "a bird in a tree," but Ss claimed to be relying mainly on covert naming of the list items during categorization.

Of the 12 FARMSCENE Ss, 10 preferred VOC to $\mathrm{V}-\mathrm{G}, 1$ had the opposite preference, and 1 had none. The respective figures for FARMSPOKEN were 4 (for VOC), 7 (for V-G), and 1 (no preference). This, too, is consistent with the trend in the output data.

\section{EXPERIMENT II}

In this experiment, visualization of FARM was encouraged by using an appropriate instructional set. The Ss were read the list and told to create a coherent mental picture made up from the words' referents. It has been claimed that such a set induces imaginal coding of verbal material (e.g., Bower, 1968). If this is true and if V-G disrupts recall of visually coded material, evidence of visual conflict should be present at recall.

\section{Method}

The material and procedure were the same as those used for the FARMSPOKEN group in Experiment I, except that the following instructions were inserted just prior to presenting $S$ with the list for acquisition: "I want you to notice that all the words in this list refer to things that you can picture in your mind. As well, the things can be put together to form an entire scene. We've found that it helps you to learn the words if you try to build up a mental picture using the things referred to in the list. Try to make a coherent picture from the whole list." The Ss were six male and six female McMaster undergraduates.

\section{Results and Discussion}

Output times were consistent with predictions. The Ss took an average of $1.6 \mathrm{sec}$ longer with V-G than with VOC, this difference being significantly greater than that for the Ss in Experiment I, who received no instructional set (FARMSPOKEN) $[\mathrm{t}(22)=2.48, \mathrm{p}<.025]$. The absolute response speeds are given in Table 1.

There were eight Ss who reported success in generating coherent mental pictures. Comments that V-G interfered with visualization at recall were again common.

The results fit the picture painted by Experiment I; an experimental manipulation said to induce visual imagery in learning led to conflict between recall of the learned material and a concurrent visually guided response. In total, it seems clear that this technique can detect internal visual representation, or at least show when Ss reach a certain level of dependence on this form of coding. Thus, one is encouraged to use it to pursue the issue raised above, the comparison of item concreteness and spatial organization as predictors of imaginal coding.

\section{EXPERIMENT III}

In this study, a new group of 12 Ss was taught the FARM list with pictorial support, but, in this case, the pictures were drawn on separate cards and presented one at a time. Thus, the concreteness of the single items was as high as it was for FARMSCENE Ss (Experiment I), but the spatial organization was missing from the stimulus material. The condition was code-named FARMSTACK.

\section{Method}

The procedure was similar to FARMSCENE, except that $S$ was shown the pictures one at a time, laid successively on top of each other during acquisition. This presentation technique was also used during the correction procedure following an incorrect recall trial.

\section{Results and Discussion}

Output times are presented in Table 1. The mean response mode difference (V.G minus VOC) of $0.6 \mathrm{sec}$ was significantly smaller than that for FARMSCENE $[\mathrm{t}(22)=3.10, \mathrm{p}<.01]$ but no different from FARMSPOKEN's $-0.2 \mathrm{sec}[\mathrm{t}(22)=0.93, \mathrm{p}>.10]$. The verbal reports from $S s$ were also closer to those of the group in Experiment I, which simply had the words read to them (FARMSPOKEN), than to FARMSCENE Ss' reports. Most said they depended on covert naming during recall for categorization, with reports of additional visual components being about as common as for FARMSPOKEN.

This comparison between FARMSCENE and FARMSTACK is the first demonstration in this series of experiments that item concreteness alone is not a good predictor of conflict between recall and a concurrent visually guided response task. It seems to have been the addition of spatial organization in the stimulus material that was crucial. 


\section{EXPERIMENT IV}

This experiment used as stimulus material a list mentioned in the Introduction: chimney roof ceiling wall carpet floor door steps path lawn. The code-name HOUSE was adopted. In pilot work all Ss to whom the list was read quickly noticed the spatial organization among the words' referents and claimed to utilize that feature during learning and recall. Thus, it was predicted that even with a verbal presentation of this list, evidence of recall-response conflict would be found, more so than with another list of equally concrete words (e.g., FARM) that lacked the obvious spatial cohesiveness of HOUSE.

\section{Method}

The 12 Ss were tested similarly to those in FARMSPOKEN (Experiment I). The $\mathrm{E}$ read the list of words to them during the acquisition phase and at no time did they receive pictorial support. The two categorization bases were soft (carpet lawn) and vertical (chimney wall door).

\section{Results and Discussion}

The prediction of visual conflict was confirmed, as the absolute speeds suggest (Table 1 ). The Ss took a mean of $1.7 \mathrm{sec}$ longer to respond when using V.G than VOC, a difference that significantly exceeded the corresponding figure for FARMSPOKEN $(-0.2 \mathrm{sec})[\mathrm{t}(22)=2.39$, $p<.025]$. Subjective reports were similar to those in FARMSCENE. All Ss but one said that they visualized a house structure and frequently commented that V-G suppressed imagery during recall. Most Ss reported strong directional components in their images. Phrases like "moving down a house" and "working down from the top of a house" were common.

\section{EXPERIMENT $V$}

In each of the cases where clear signs of recall-response conflict were found (FARMSCENE and HOUSE), the individual items formed parts of a meaningful whole as well as (according to S's reports) being distributed in imaginal space. The question asked in the present experiment was this: Is this figural unity necessary before Ss are induced to rely sufficiently on imagery to produce visual conflict or will any arbitrary series of items suffice, as long as the items are spatially organized.

Method

\section{Material}

The following noun list was generated: pig trunk dog fire wheat child bale insect ocean tulip. No pilot $S$ saw any overall theme uniting the words' referents. The matris of drawings shown in Fig. 4 was prepared and the matellal was code-named PIGMATRIX. Categorization bases were plant and plant matcrial (trunk wheat bale nilip) and ammal (pig dog child insert)

\section{Procedure}

The experiment was conducted in the same favion w

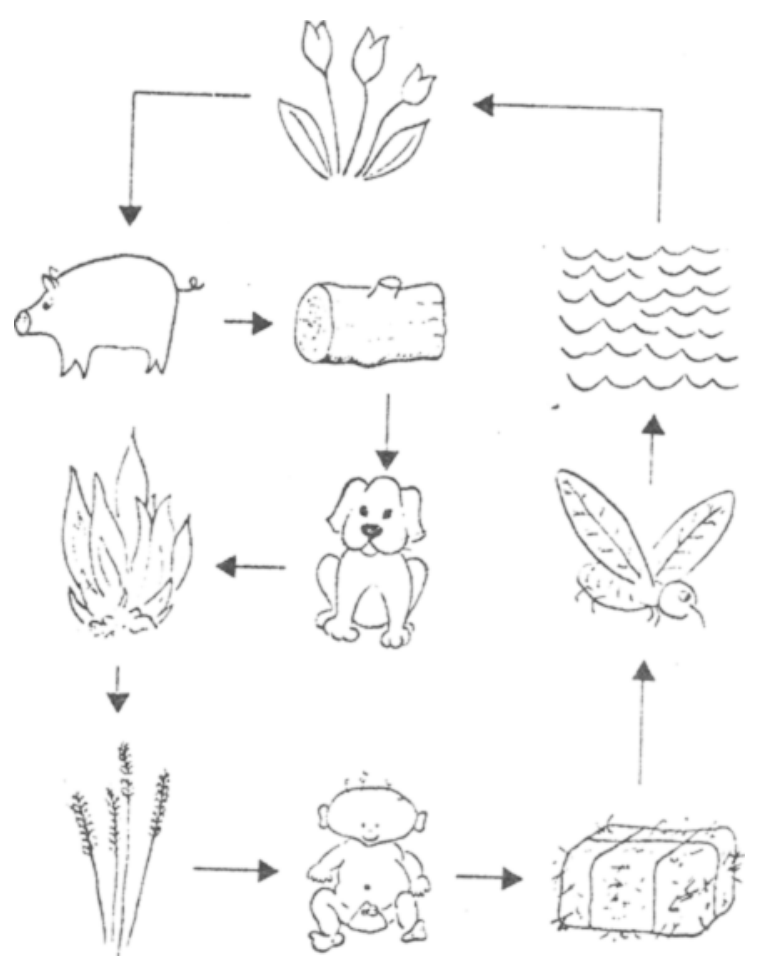

Fig. 4. Picture matrix for PIGMATRIX.

IAARMSCENE (Experiment I). The 12 Ss learned the list with the aid of the picture matrix.

\section{Results and Discussion}

Output times are in Table 1. The mean difference (V-G minus VOC) was $4.4 \mathrm{sec}$, with every $\mathrm{S}$ showing this directional difference. The way Ss described their own performance fitted the general pattern that emerged in some earlier conditions-reported reliance on visual imagery plus claims that V-G interrupted that form of internal representation. The directional component reported in some previous conditions was present quite clearly in this experiment; some Ss made noticeable finger or head and eye movements that traced out the matrix while they were recalling the items for categorization.

Since the mean output-mode difference for FARMSCENE was $3.2 \mathrm{sec}$, the hypothesis that PIGMATRIX (4.4-sec difference) would lead to significantly less visual conflict than a spatial display of pictures that formed a meaningful visual-spatial whole must be rejected. It appears. therefore. that spatial differentiation of items is sufficient to generate a recall strategy that conflicts with a concurrent visually guided response. It is possible, therefore, that scene-like presentations produce visual conflict at recall because the items are stored in a spatial scheme.

\section{EXPERIMENT VI}

Evidence to date suggests that visual conflict is better predicted by spatial organization than by item concreteness. In this final experiment. that proposition 


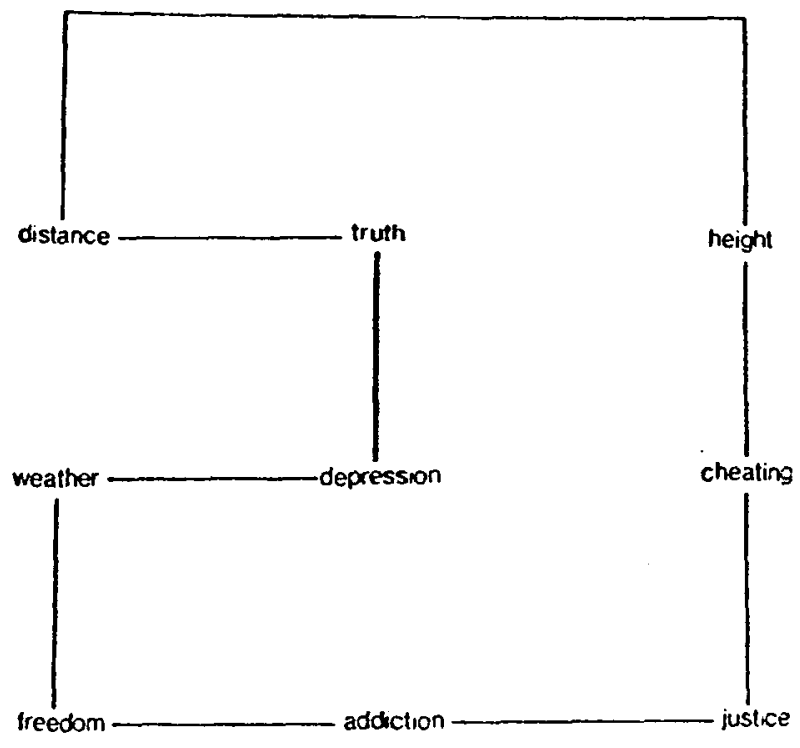

Fig. 5. Matrix of ABSTRACT WORDS.

is subjected to a more radical test by having Ss learn and subsequently categorize a list of abstract words presented in a spatial matrix. Abstract words, even presented visually, are traditionally regarded as low in concreteness.

\section{Method}

Figure 5 shows the stimulus material used. Only nine items were included because of the extra learning difficulty usually associated with abstract material. The categorization bases were good (truth freedom justice) and bad (depression addiction cheating).

The procedure was similar to that for PIGMATRIX, in that Ss were shown the matrix during the learning phase. As usual, $12 \mathrm{Ss}$ were used.

\section{Results and Discussion}

Table 1 contains the output times. The mean response-mode difference was $2.9 \mathrm{sec}$, close to the $3.2 \mathrm{sec}$ for FARMSCENE and not even significantly less than the figure for PIGMATRIX $[4.4, \mathrm{t}(22)=1.24$, $p>05]$. Here are some typical subjective reports: "I had a graphical structure in mind." "I ran round the list with my eyes-for speaking. I couldn't see it as well for pointing because I was staring at this (output sheet)." "The shape helped-I thought of the location if I got stuck in remembering the word." The results supported the contention that visual conflict occurs when the items are arranged spatially and is relatively independent of item concreteness.

\section{GENERAL DISCUSSION}

The consistency of the results across a variety of stimulus material permits one to reach conclusions with a fair degree of confidence. First, a visually guided response does seem to disrupt recall of a list of items learned under conditions regarded as likely to induce mediating imagery (Experiments I and II). Second, this visual conflict is most in evidence when the items are spatially organized and seems largely independent of item concreteness. It is argued, therefore, that the presence or absence of spatial organization is an important characteristic of stimulus material when it comes to predicting whether the material will be coded imaginally and needs to be considered separately from item concreteness. It should be noted that this spatial quality may be embodied in the words' referents (as in HOUSE) or physically present in the stimulus material (as in PIGMATRIX). One can speculate that in both cases the recall process involves a scan of imaginal space.

Failure to find that visual conflict is controlled by item concreteness deserves comment, because the relative ease with which high-concrete words are learned and recalled has usually been interpreted as an imagery effect. Why, then, does high item concreteness not generate visual conflict in the present task?

A possible answer is that it is only when material is spatially organized that useful imagery is evoked for tasks demanding sequential output, as the present ones do. This accords with the opinion that visual imagery “. . . is not specialized for serial processing unless linked to an integrated (symbolic) motor response system, such as might be involved in imaginally tracing the outline of a block letter ... or imagining oneself moving along a familiar route containing sequentially arranged 'signposts' [Paivio, 1971, p. 180]." Perhaps mentally tracing a path through the FARM scene, down the HOUSE, or around a matrix falls into the same class as the above examples. In the absence of such spatial relationships among the items, Ss might use the verbal system "... assumed to be specialized for serial or sequential processing by virtue of the temporal nature of the auditory-motor speech system [Paivio, 1971, p. 180]. Lashley, too, has drawn attention to the "... interaction of temporal and spatial systems .... Spatial and temporal order ... appear to be almost completely interchangeable in cerebral action .... when the memory trace is formed it is integrated with the directional characters of the space system, which give it position in reference to other associated traces .... the assumption is that these space characters can be scanned by some other level of the coordinating system and so transformed into succession [1951. pp. 128-129]." Thus, using a task requiring serial recall may stack the cards in favor of finding conflict only with material in which items are linked spatially (irrespective of concreteness), because it may be only then that $S$ generates useful visual imagery.

Two questions about imagery in nonserial tasks are raised at this point. One is whether there is evidence for the direct involvement of the visual system in tasks lacking the kind of spatial quality of the present material. The answer seems a clear "yes." Segal and Fusella (1970) present data that can be interpreted as showing competition between perception and acts of imagination, and Atwood (1971) demonstrated similar conflict in a paired-associate learning task. (Some 
difficulty has been experienced in attempting to replicate Atwood's results, suggesting perhaps that a fairly fragile phenomenon is under investigation; see Paivio, 1971, pp. 372-374).

The second question that needs to be asked is whether item concreteness is the only predictor of performance in nonserial tasks in which imagery is a likely component process. One might be tempted to say that spatial relatedness does add something, based on evidence like that of Epstein. Rock, and Zuckerman (1960). They showed that picture pairs presented as meaningful units (e.g., a hand inside a bowl) were better learned than the same two pictures drawn separately. although in both cases the concreteness of the individual items was the same (cf. FARMSCENE vs FARMSTACK in the present series). But can we be sure that visual imagery was involved? Analogous improvements in performance were obtained by changing relatedness of noun pairs, i.e., by giving a pair like "hand or bowl" vs "hand in bowl." Furthermore, Bobrow and Easton (1972) have confirmed that it is relational organization and not mere spatial proximity that is the crucial facilitating characteristic. Thus, while it is true that item concreteness is not the whole story, it is not clear whether the additional component process is an imaginal one nor, even if it is. whether the term "spatial" is the appropriate adjective to describe it. Possibly the technique used in this research, that of looking for conflict between mental processes and perceptual activity, could be used to throw light on this issue.

\section{Explanations for Conflict in These Experiments}

Conflict may occur for one or both of the following reasons: The V.G response suppresses imagery of the individual items or (assuming the act of recall involves a spatial scan) it interferes with the imagined scan when getting from one location to the next. This latter possibility is interesting in light of the suggestion that performing a sequential scan of information distributed in imaginal space brings into play mechanisms that would be used in an actual examination of the (physically present) information. Berlyne (1965). for example, suggests that, if a person is asked to name the States of the U.S.A. in a line from California to New York City, he would most likely do it in stages by successively imagining areas in a west to east direction and that the "... stages will be linked to one another by processes that are clearly equivalent to the eye movements ... that he would have used if he had been examining an actual map of the United States and reading off the names of the states from it [p. 142]." Following that line of reasoning. in the experiments reported here conflict may have arisen because the (motor) mechanism underlying the use of the imagined. spatially distributed information was also requised in responding to the spatially distributed output sheet. with the cover! and overt distributions having different directional characteristics. In other words. Ss may have had trouble thinking in one direction and directing movement in another, because both orienting responses utilized the same equipment. In fact, various experiments aimed at testing the notion that visual conflict in this experimental situation springs largely from incompatible covert and overt orienting activities have been conducted in our laboratories. The results support that view.

Finally, it should be noted that the present evidence relates mostly to retrieval processes and leaves acquisition largely untouched. It would be interesting to see if evidence exists for the direct involvement of the visual system during the learning phase, evidence that is consistent with these results. It might be predicted, for example, that learning a list like HOUSE would be made difficult if a visual task were performed during acquisition. A less spatially constrained list, such as FARM. should be less affected.

\section{REFERENCES}

Atwood, G. E. An experimental study of visual information and memory. Cognitive Psychology. 1971. 2. 290-299.

Berlyne. D. E. Structure and direction in thinking. New York: Wiley. 1965.

Bobrow. S. A., \& Easton. R. D. A confirmation that relational organization facilitates memory. Psychonomic Science, 1972. 29. 256-257.

Bower, G. H. Mental imagery and associative learning. Paper read at Fifth Carnegie-Mellon Symposium on Cognition. April 1968.

Brooks. L. R. The suppression of isualization by reading. Quarterly Journal of Experimental Psychology: 1967, 19. 289-299.

Brooks. L. R. Spatial and verbal components of the act of recall. Canadian Journal of Psychology. 1968. 22. 349-368.

Brook: L. R. An extension of the conflict between visualization and reading. Quarterly Journal of Experimental Psychology, 1970. 22, 91-96.

Epstein. W.. Rock. I.. \& Zuckerman. C. B. Meaning and familiarity in associative learning. Psychological Monographs. 1960.74(4. Whole No. 491).

Kintsch. W. Abstract nouns: Imagery versus levical complexity. Journal of Verbal Learning \& Verbal Bchavior. 1972. 11. $59-65$

Lashley, K. S. The problem of serial order in behavior. In L. A. Jeffress (Fd.1. Cerebral mechanisms in behavior: The Hixon symposium. Neu York: W'iley. 1951.

Neisser. ('. Vinual imagery as process and as experience. Paper presented at the Center for Research in Cognition and Affect. New York. June 1968.

Paivio. A. Imagery and rerhal processes. New York: Holt. Rinehart. \& Winston. 1971.

Segal. S. J.. \& I usella. V. Intluence of imaged pictures and sounds on detection of visual and auditory signals. Journal of Fxperimental Psychology. 1970. 83.458-464

Shepard. R. N. Learning and recall as organization and search. Journal of Verbal learning \& Verbal Behavior, 1966. 5. 201-204.

Skinner. B. 1. Scionce and human beharior. Neu York: Mac Millan. 1953.

(Received for publication A pri! 2. 1973 : revioion received Na! $7.1973,1$ 